\title{
Using ion beams for creation of nanostructures on the surface of high-stable materials
}

\author{
I.V. Gorbov, V.V. Petrov, A.A. Kryuchyn \\ Institute for Information Recording, NAS of Ukraine (IPRI NASU) \\ 2, M. Shpak str., 03113 Kyiv, Ukraine \\ ${ }^{1}$ Phone: (38-044) 454-22-09, fax: (38-044) 241-72-33, e-mail: ivan-gorbov@list.ru \\ ${ }^{2}$ Phone: (38-044) 454-21-51, fax: (38-044) 241-72-33, e-mail: petrov@ipri.kiev.ua \\ ${ }^{3}$ Phone: (38-044) 454-21-52, fax: (38-044) 241-72-33, e-mail: ipri@ipri.kiev.ua
}

\begin{abstract}
Main ion-beam etching techniques for creation of nanostructures on the surface of high-stable materials have been considered. Methods of information recording in the form of nanostructure on the metallic substrate surface have been analyzed. Application of glass substrate for creation long-term data carrier was proposed. Microrelief information record on the glass substrate surface was obtained using the ionbeam etching.
\end{abstract}

Keywords: dry etching technique, ion-beam etching, reactive ion-beam etching, highstable material, nanostructure, glass substrate, long-term data carrier.

Manuscript received 31.10.06; accepted for publication 26.03.07; published online 01.06.07.

\section{Introduction}

Micro- and nano-structuring the surfaces is important for the production of many components and systems such as gratings, diffractive optical elements, data carriers, planar wave-guide devices. Although wet etching is well developed for some of these applications, it has inherent limitations caused by undercutting of mask materials, especially for micron and submicron pattern sizes [1]. Dry etching techniques, on the other hand, can generate anisotropic etch profiles and for this reason have come into favour.

The performance of recorded information in the form of microrelief structure on the high-stable material substrate surface is one of the basic approaches for the long-term data storage. Norsam Technologies Inc. realizes data recording on the nickel substrate surface in analog and digital form [2]. The same data carrier is reliable but very expensive. Employment of inexpensive material (quartz, sapphire, silicon, glass) is more appropriate in most cases of archive storage.

\section{Analysis of ion-beam etching technique}

Most usually nanostructure creation on the substrate surface is realized by the etching via a protective contact mask with preformed image. The contact mask image size and type of the etching define dimensions of nanostructures. The masks can be classified by three classes: organic polymer film masks (photoresist, electron-beam resist and ion-beam resist), metal film masks and inorganic or dielectric film masks (carbon, silicon dioxide and nitride) [3]. Using the electron-beam and ion-beam resists allows to create a preformed mask image with $50 \mathrm{~nm}$ resolution [4].

Data recording density increase causes a reduction of the structure characteristic dimensions, for example, a track width of Blue-Ray Disc is $150 \mathrm{~nm}$. The methods of etching with a highest resolution are those with using the focused ion beams, especially ion beam etching and reactive ion beam etching. The ion beam etching implicates substrate surface layer removal by means of physical material sputtering by the inert gas energetic ions that are chemically passive to the substrate materials.

The mechanism of material removal in reactive ion beam etching is rather due to chemical reaction between reactive particles and the substrate material atoms than physical sputtering [5]. Although many researchers recognize that these two mechanisms are synergistic, i.e. the bombardment catalyzes the surface chemical reactions. Thus, this method of etching is also called chemically assisted ion beam etching.

But although widely developed for silicon dioxide (on silicon), as well as to silica (quartz) glass substrates, there has been limited study of multicomponent silicate 
or phosphate glass. In the case of silicon dioxide (silica), $\mathrm{CF}_{4}$ and/or $\mathrm{CHF}_{3}$ are commonly used dry etching gases because they generate highly volatile $\mathrm{SiF}_{4}, \mathrm{CO} / \mathrm{CO}_{2}$ and $\mathrm{H}_{2} \mathrm{O}$ reaction products. Due to [1] the net reaction in $\mathrm{CF}_{4}$, for example, is

$$
\mathrm{SiO}_{2}+\mathrm{CF}_{4} \stackrel{\mathrm{e}^{-}}{\longrightarrow} \mathrm{SiF}_{4}+\mathrm{CO}_{2} \text {. }
$$

\section{Experiments and results}

The data layer in the form of micropits was obtained on the glass substrate surface using ion beam etching (Fig. 1). The method of data recording is shown in Fig. 2. On the glass substrate with a positive photoresist layer, the data recording laser beam was focused. The ion-beam etching was performed via mask windows that appeared in the photoresist layer after selective etching.

The photoresist film was firmly adherent to the glass substrates and could not be removed in acetone or the commercial striping solution after the etching process (presumably, due to some bombardment or electric field induced interfaces reactions). Thus, it was necessary to use $\mathrm{O}_{2}$ plasma to remove the residual photoresist.

The scheme of ion beam etching system is shown in Fig. 3. The gas source consists of two collars $(8,9)$ with bore on the top side. It is under dc voltage $2 \mathrm{kV}$. Inert gas (Ar) comes into it through the insulator 10 . Plasma is generated by glow discharge in the space between the gas source and grounded electrode 7 . The ion beam is extracted from it by means of electrostatic field and focused using the coil 6 .

Ion beam consists of positive charge particles 5 that cause formation of the surface charge on the working part of the surface 1. It is insufficient for the ion beam etching of the metal substrate, because a surface charge drains to grounded body. But stored surface charge inhibits further etching of the dielectric working part. Therefore, we used the tungsten filament 2 as an electron emitter to form an electron cloud 3 for creation of neutralized ion beam 4 .

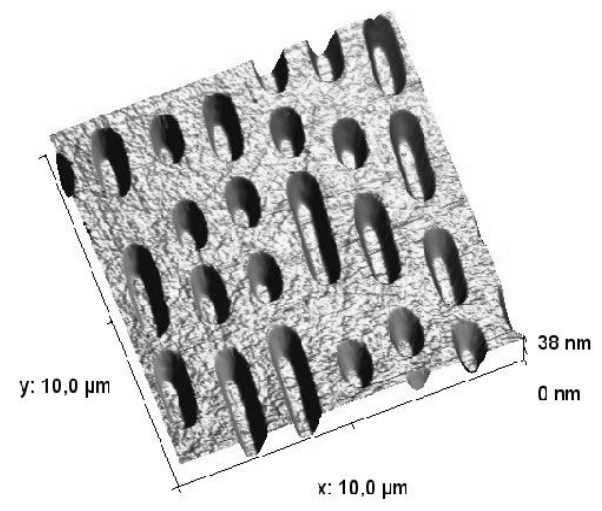

Fig. 1. Microrelief structure of the data layer on the glass substrate surface.

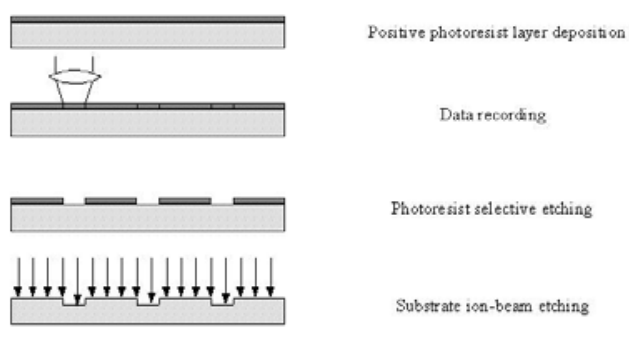

Fig. 2. Data layer creation on the high-stable substrate.

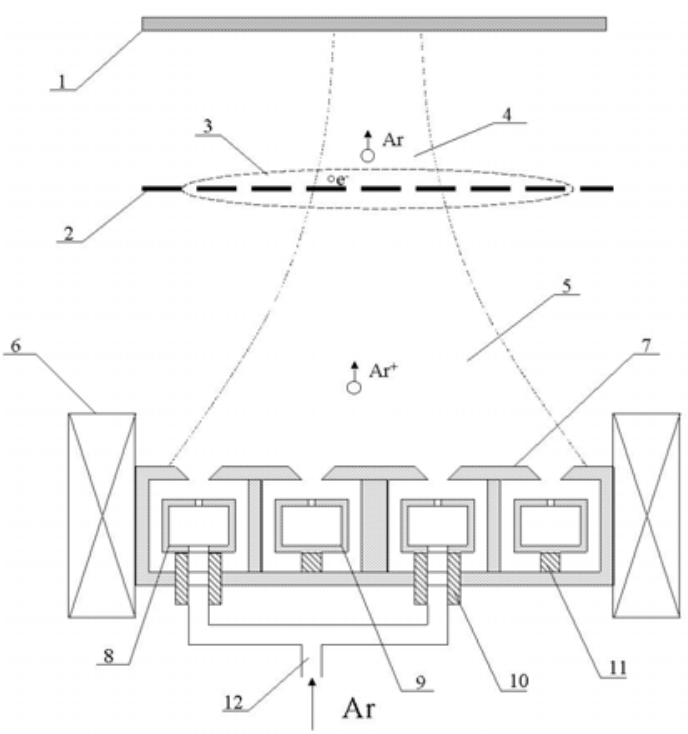

Fig. 3. The scheme of the ion beam etching system: 1 working part; 2 - electron emitter (tungsten filament); 3 electron cloud; 4 - neutralized ion beam; 5 - ion beam; 6 focusing coil; 7 - grounded electrode; 8 - alive external gas source; 9 - alive internal gas source; 10 - insulator with gas input; 11 - insulator; 12 - gas input.

\section{Conclusions}

Using the ion beam allows to create a nanostructure on the high-stable material surface. Nanostructure dimensions are defined by material masks and image record methods. The ion-beam (and reactive ion-beam) etching can be used for creation of long-term data carrier. The mentioned data recording method is appropriable for other high-stable material substrates. It is necessary to select a proper protective contact mask, operating gas mixture and etching regime.

\section{Acknowledgments}

The authors thank the staff of V. Lashkaryov Institute of Semiconductor Physics of NAS of Ukraine for their assistance in nanostructure measurements. 


\section{References}

1. E. Metwalli, C.G. Pantano, Reactive ion etching of glasses: composition dependence // Nucl. Instrum. Meth. in Phys. Research. B 207, p. 21-27 (2003).

2. Norsam Technologies Inc. http://norsam.com/ hdrosetta.htm.

3. B.S. Danilin, V.Yu. Kireyev, Ion etching of microstructures. Sov. Radio, Moscow, 1979 (in Russian).
4. A. Hohenau, H. Ditlbacher, B. Lamprecht, J.R. Krenn, A. Leitner, F.R. Aussenegg, Electron beam lithography, a helpful tool for nanooptics // Microelectronic Engineering 83, p. 1464-1467 (2006).

5. B.S. Danilin, V.Yu. Kireev, Application the lowtemperature plasma for etching and cleaning of materials. Energoatomizdat, Moscow, 1987 (in Russian). 